12th International Symposium on Cosmology and

Particle Astrophysics (CosPA 2015)

International Journal of Modern Physics: Conference Series

Vol. 43 (2016) 1660204 (7 pages)

(C) The Author(s)

DOI: $10.1142 / \mathrm{S} 2010194516602040$

\title{
Suppression of the Primordial Gravitational Waves
}

\author{
Gansukh Tumurtushaa \\ Department of Physics, Sogang University, Seoul 04107, Korea \\ gansuh@sogang.ac.kr \\ Seoktae Koh \\ Department of Science Education, Jeju National University, Jeju 63243, Korea \\ kundol.koh@jejunu.ac.kr \\ Bum-Hoon Lee \\ Department of Physics, Sogang University, Seoul 04107, Korea \\ Center for Quantum Spacetime, Sogang University, Seoul 04107, Korea \\ Asian Pacific Center for Theoretical Physics, Pohang 37673, Korea \\ bhl@sogang.ac.kr
}

Published 7 July 2016

\begin{abstract}
We study the primordial gravitational waves induced by space-space condensate inflation model. For modes that cross the comoving horizon during matter dominated era, we calculate the energy spectrum of gravitational waves. The energy spectrum of gravitational waves for our model has significantly suppressed in the low frequency range. The suppression occurs due to the phase transition during the early evolution of the Universe and depends on model parameter.
\end{abstract}

Keywords: Inflation; primordial gravitational waves.

\section{Introduction}

Inflation paradigm, an early period of accelerated expansion, is the leading candidate of explaining the primordial Universe ${ }^{1-3}$ As a result, inflation models predict that this acceleration expansion would create ripples in the curvature of spacetime, generating gravitational waves, that would remain energetic enough to leave an imprint on the Cosmic Microwave Background(CMB) radiation, approximately 380,000 years later ${ }^{4-} .6$ The nearly scale-invariant, adiabatic, and almost Gaussian primordial curvature perturbation predicted by slow-roll inflation is consistent with the recent observations by Planck $^{7}$ while the primordial tensor perturbation, also

This is an Open Access article published by World Scientific Publishing Company. It is distributed under the terms of the Creative Commons Attribution 4.0 (CC-BY) License. Further distribution of this work is permitted, provided the original work is properly cited. 
known as the primordial gravitational waves, is still on the way to be observationally detected. ${ }^{8}$ The observational detection of primordial gravitational waves would not only verify a success of inflation, but would also open a new window to understand the physics of the early Universe.

We calculate the energy spectrum of primordial gravitational in inflation with nonlinear sigma fields ${ }^{9-11}$ having $\sigma^{a} \sim x^{a}$ waves for the modes that re-entered the comoving horizon during the matter dominated era. It is assumed that the phase transition between two consecutive regimes to be abrupt. In such case, the cosmic expansion is the only damping effect after modes cross the comoving horizon.

The outline of our paper is the following. The basic setup is introduced in Section 2 while main calculation of the energy spectrum of primordial gravitational waves generated by the space-condensate inflation is provided in Section 3. In section 4 , we conclude our results.

\section{Setup}

In this section, we construct the background setup for space-condensate inflation model $^{9-11}$ which is considered as a source to the primordial gravitational waves in this work. We start with the following action,

$$
\mathcal{S}=\int d^{4} x \sqrt{-g}\left[\frac{1}{2 \kappa^{2}} R-\frac{1}{2} g^{\mu \nu} \partial_{\mu} \phi \partial_{\nu} \phi-\frac{1}{2} g^{\mu \nu} \delta_{a b} \partial_{\mu} \sigma^{a} \partial_{\nu} \sigma^{b}-V(\phi)\right],
$$

where we have an additional kinetic term for the triad of scalar fields, $\sigma^{a}$, in addition to action for scalar field, $\phi$. To construct the spatial-condensate inflation model, we consider the flat FLRW metric, $d s^{2}=-d t^{2}+a(t)^{2}\left(d x^{2}+d y^{2}+d z^{2}\right)$, along with the spatially linear configuration for $\sigma$ fields, $\sigma^{a}=\xi x^{a}$ where $\xi$ is the arbitrary constant. Thus, the background equations of motion are obtained as

$$
\begin{aligned}
H^{2} & =\frac{1}{3 M_{p}^{2}}\left(\frac{1}{2} \dot{\phi}^{2}+V+\frac{3 \xi^{2}}{2 a^{2}}\right), \\
0 & =\ddot{\phi}+3 H \dot{\phi}+V_{\phi} .
\end{aligned}
$$

If $\xi$-term in Eq. (2) dominates over both potential and kinetic terms, then we obtain constant comoving horizon, ${ }^{17}$

$$
(a H)^{-1} \simeq\left(\frac{\xi^{2}}{2 M_{p}^{2}}\right)^{-\frac{1}{2}}
$$

during the early evolution of the Universe. On the other hand, as the scale factor in Eq. (2) increases exponentially during inflation, the effect of $\xi$-term decreases rapidly such that at the end inflation our model asymptotically approaches to the standard single field inflation model with the negligible small contribution from $\xi$-term.

Therefore, there exist the early phase transition during early evolution of the Universe and usual slow-roll inflation takes place after the phase transition. 


\section{The primordial gravitational waves signature}

The gravitational waves are characterized by the tensor perturbation, $h_{i j}$, of the flat FLRW metric ${ }^{18-}, 20$

$$
d s^{2}=a^{2}\left[-d \tau^{2}+\left(\delta_{i j}+h_{i j}\right) d x^{i} d x^{j}\right]
$$

where $\delta_{i j}$ is the flat space metric and $h_{i j} \ll \delta_{i j}$. The conformal time $\tau$ relates to the cosmic time $t$ via scale factor $a$, and it is $d \tau=d t / a$. With this metric, we expand Eq. (1) in second order in $h_{i j}$ and obtain

$$
S^{(2)}=\int d \tau d^{3} x \frac{a^{2}}{8 \kappa^{2}}\left(h_{i j}^{\prime 2}+h_{i j} \partial_{k} \partial_{k} h_{i j}-2 \kappa^{2} \xi^{2} h_{i j} h_{j i}\right)
$$

where $h_{i i}=0=\partial_{i} h_{i j}$. The power spectrum of primordial gravitational waves is calculated as follows. The tensor perturbation, $h_{i j}$, can be transformed in the Fourier space as

$$
h_{i j}(\tau, \mathbf{x})=\frac{\sqrt{2}}{M_{p}} \sum_{\lambda} \int \frac{d \mathbf{k}}{(2 \pi)^{3 / 2}} \epsilon_{i j}^{(\lambda)}(\mathbf{k}) h_{\lambda, \mathbf{k}} e^{i \mathbf{k x}}
$$

where superscript $\lambda$ denotes each polarization states of the tensor perturbation and $\epsilon_{i j}^{(\lambda)}$ is the polarization tensors which satisfy $k_{j} \epsilon_{i j}^{(\lambda)}(\mathbf{k})=0, \epsilon_{i i}^{(\lambda)}(\mathbf{k})=0$, and $\epsilon_{i j}^{(\lambda)}(\mathbf{k}) \epsilon^{i j\left(\lambda^{\prime}\right)}(\mathbf{k})=\delta^{\lambda \lambda^{\prime}}$.

Thus we have the linearized equation of motion for tensor perturbations in Einstein gravity is ${ }^{17}$

$$
h_{\lambda, k}^{\prime \prime}+2 \mathcal{H} h_{\lambda, k}^{\prime}+\left(k^{2}+\frac{2 \xi^{2}}{M_{p}^{2}}\right) h_{\lambda, k}=0,
$$

where $\mathcal{H}=a^{\prime} / a$ and ${ }^{\prime} \equiv d / d \tau$. The mode solutions of Eq. (8) evolve as constant before they cross the horizon $(k \ll a H)$; then they damp, as $h_{\lambda, k} \sim 1 / a$, well inside the horizon $(k \gg a H)$. As we mentioned in previous section that $\xi$-term would be negligible small at the end of inflation, therefore, the effect of $\xi$-term in Eq. (8) is also negligible.

The strength of the primordial gravitational waves is characterized by their energy spectrum ${ }^{18-20}$

$$
h_{0}^{2} \Omega_{G W}(k)=\frac{h_{0}^{2} k^{2}}{12 H_{0}^{2}} \mathcal{T}^{2}(k) \mathcal{P}_{T}(k),
$$

where $H_{0}$ is the present value of the Hubble parameter, $H_{0}=100 h_{0} \mathrm{kms}^{-1} \mathrm{Mpc}^{-1}$, and $h_{0}$ is dimensionless Hubble parameter. Transfer function, $\mathcal{T}$, reflects the damping effect for the modes inside the Hubble horizon. Therefore, it can be calculated from Eq. (8) and good fit to the transfer function for the modes well inside the horizon is $21-22$

$$
\mathcal{T}(k)=\frac{3}{k^{2} \tau_{0}^{2}} \frac{\Omega_{m}}{\Omega_{\Lambda}} \sqrt{1+\frac{4}{3}\left(\frac{k}{k_{e q}}\right)+\frac{5}{2}\left(\frac{k}{k_{e q}}\right)^{2}},
$$

where $k_{e q}$ is the wavenumber when matter and radiation have equal energy density, $k_{e q}=0.073 \Omega_{m} h_{0}^{2} \mathrm{Mpc}^{-1}$ where $h_{0}$ is the dimensionless Hubble parameter, $\tau_{0}$ is the 
present conformal time, and $\Omega_{m}$ and $\Omega_{\Lambda}$ are the present energy density fractions of the matter and the vacuum, respectively. For modes re-entered the horizon during the matter dominated era, Eq. (10) evolves as $\mathcal{T}(k) \sim k^{-2}$.

The inflationary power spectrum, $\mathcal{P}_{T}(k)$, of tensor modes in Eq. (9) is assumed to be power-law form as

$$
\mathcal{P}_{T}(k)=\mathcal{P}_{T}\left(k_{*}\right)\left(\frac{k}{k_{*}}\right)^{n_{T}+\frac{\alpha_{T}}{2} \ln \left(k / k_{*}\right)},
$$

where $\alpha_{T} \equiv d n_{T} / d \ln k$ is the running of tensor spectral index $n_{T}$ and $\mathcal{P}_{T}\left(k_{*}\right)$ is the amplitude of tensor power spectrum at the pivot scale $k_{*}$. The amplitude of tensor power spectrum, $\mathcal{P}_{T}\left(k_{*}\right)$, can relate to that of the scalar power spectrum, $\mathcal{P}_{S}\left(k_{*}\right)$, which is very well constrained by observational data ${ }^{7}$ via tensor-to-scalar ratio, $r$, which is another observable parameter as $\mathcal{P}_{T}\left(k_{*}\right) \equiv r \mathcal{P}_{S}\left(k_{*}\right)$.

The observable quantities in Eq. (11) for the space-condensate inflation model are obtained in Ref. 10 as follows

$$
n_{T} \simeq-2 \epsilon_{V}+\frac{31 \xi^{2}}{6 k^{2} M_{p}^{2}}, \quad \alpha_{T}=-\frac{31 \xi^{2}}{3 k^{2} M_{p}^{2}}, \quad r \simeq 16 \epsilon_{V}+\frac{16 \xi^{2}}{k^{2} M_{p}^{2}} .
$$

where $\epsilon_{V}$ and $\eta_{V}$ are the potential based slow-roll parameters ${ }^{\text {a }}$.

Therefore, by substituting Eqs. (10)-(12) into Eq. (9), we obtain the energy spectrum of the primordial gravitational waves for modes that cross the horizon during matter dominated era,

$$
h_{0}^{2} \Omega_{G W}=\frac{3 h_{0}^{2} \mathcal{P}_{S}\left(k_{*}\right)}{16 \pi^{2} H_{0}^{2} \tau_{0}^{4}}\left(\frac{\Omega_{m}}{\Omega_{\Lambda}}\right)^{2} \frac{r}{f^{2}}\left(\frac{f}{f_{*}}\right)^{-\frac{r}{8}+\frac{43 \xi^{2}}{24 \pi^{2} M_{p}^{2} f^{2}}-\frac{31 \xi^{2}}{24 \pi^{2} M_{p}^{2} f^{2}} \ln \left(\frac{f}{f_{*}}\right)},
$$

where we consider $k \ll k_{e q}$ in Eq. (10) and $f$ is the physical frequency observed today and defined as $f \equiv k / 2 \pi$, while $f_{*}$ is the reference frequency $f_{*} \equiv k_{*} / 2 \pi$.

In our numerical plots below, we use the following cosmological parameters, provided by Ref. 7 , where $\Omega_{m}=0.315, \Omega_{\Lambda}=0.685, \mathcal{P}_{S}\left(k_{*}\right) \simeq 2.19 \times 10^{-9}, k_{*}=$ $0.002 \mathrm{Mpc}^{-1}, h_{0} \simeq 0.6731$, and $\tau_{0}=1.41 \times 10^{4} \mathrm{Mpc}$.

In Fig. 1, we plot Eq. (13) as function of $f$ for fixed $r$. We set $r=10^{-1}$ in Fig. 1 because recent observational data ${ }^{7}$ predicts $r_{0.002}<0.11$ as a upper bound for $r$. For nonzero value of $\xi$ one can see that the energy spectrum of the primordial gravitational waves suppresses. As we increase the value of $\xi$ parameter from smaller to larger, $\xi=4 \times 10^{-20}$ (dashed), $\xi=10^{-19}$ (dot-dashed), and $\xi=4 \times 10^{-19}$ (dotted), the we see even more suppression.

In Fig. 2, we plot Eq. (13), but this time we fix $\xi$ parameter and change the value of $r$ to see its effect to the energy spectrum. We set $\xi=0$ for solid line and $\xi=10^{-19}$ for dot-dashed line, then change $r$ value $r=10^{-1}, r=10^{-2}$, and $r=10^{-3}$, respectively from top to bottom. As seen in Fig. 2 that the change in

${ }^{\mathrm{a}}$ see Ref. 10 for details. 


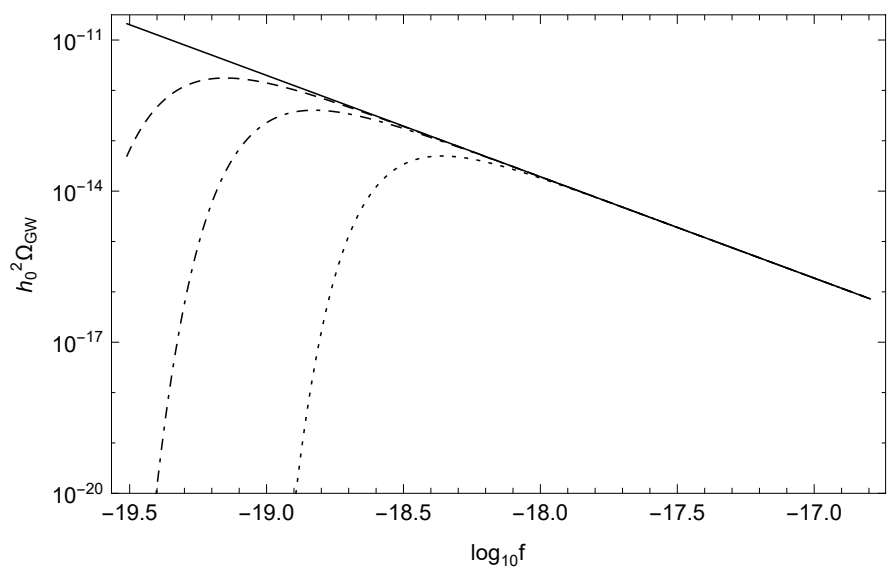

Fig. 1. The plot of Eq. (13) for fixed $r=0.1$. Four difference values of $\xi$ is considered; $\xi=0$ (solid), $\xi=4 \times 10^{-20}$ (dashed), $\xi=10^{-19}$ (dot-dashed), and $\xi=4 \times 10^{-19}$ (dotted).

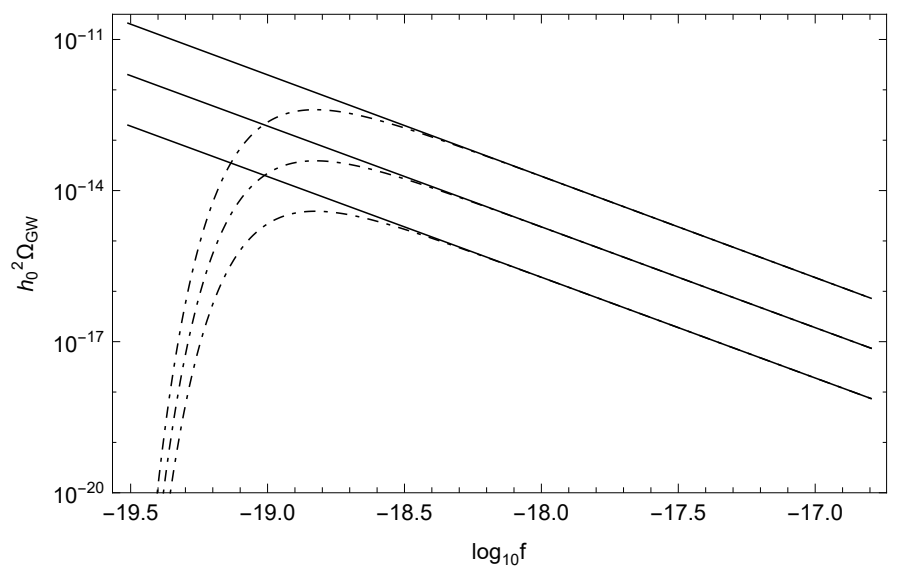

Fig. 2. The plot of Eq. (13) for fixed $\xi=0$ (solid) and $\xi=10^{-19}$ (dot-dashed). Three different values of $r$ is plotted from top to bottom; $r=10^{-1}, r=10^{-2}$, and $r=10^{-3}$, respectively.

$r$ value does not have a any influence to the suppression of the energy spectrum. Instead it only affects the amplitude of the energy spectrum.

Therefore, one can see that the suppression of the energy spectrum is depends only on model parameter $\xi$. In order to realize the suppression of energy spectrum of the primordial gravitational waves induced by the space-condensate inflation model, we need to understand the phase transition during early evolution of the Universe. ${ }^{11}$ The phase transition occurs when $V \sim 3 \xi / 2 a^{2}$. Before the phase transition, comoving horizon remains constant, $(a H)^{-1} \simeq\left(\xi / \sqrt{2} M_{p}\right)^{-1}$; after the phase transition, the potential energy of the Universe dominates so that the canonical slow-roll inflation takes a place. 
The existence of constant comoving horizon means that there also exists the minimum for comoving wavenumber. ${ }^{10}$ Therefore observational lower bound for the comoving wavenumber is $k_{\text {min }} \simeq \xi / \sqrt{2} M_{p}$. The modes satisfying $k \geq \xi / \sqrt{2} M_{p}$ can only cross the comoving horizon during the early phase transition, and the modes that satisfy $k<\xi / \sqrt{2} M_{p}$ never cross the comoving horizon and stay outside the horizon.

\section{Conclusion}

In this work, we have studied the primordial gravitational waves from the spacecondensate inflation model. The energy spectrum of gravitational waves for the modes that re-entered the horizon during matter dominated era is obtained in Eq. (13). Therefore, our result is true only for the modes crosses the horizon during matter dominated era or stay well inside comoving horizon.

In Fig. 1 and Fig. 2, we plot Eq. (13) as a function of frequency. The figures show that the energy spectrum of primordial gravitational waves has the significant suppression that occurs only for the non-zero values of model the parameter. The tensor-to-scalar ratio has nothing to with the suppression effect instead it does affect to the amplitude of the energy spectrum.

To realize the suppression of gravitational-waves energy spectrum, we have discussed the feature of the model that we consider. The feature of the model is that there exists the phase transition during the early evolution of the Universe which followed by the canonical slow-roll inflation. The existence of this phase transition leads to the existence the observational lower bound for the comoving wavenumber, $k_{\min } \simeq \xi / \sqrt{2} M_{p}$. The modes with $k<k_{\min }$, would never cross the comoving horizon, and stay outside the horizon. Since those modes causally disconnected to our Universe they suppress the energy spectrum of the primordial gravitational waves.

If the energy spectrum of primordial gravitation waves suppresses in the lowfrequency range, it is then expected that such suppression should be imprinted in the CBM measurement. Therefore, in our next project, we would like to see effects of model parameter to the CMB angular power spectrum for BB-mode and to generalize of current result to the modes that cross the coming horizon during radiation dominated era and still stay well inside the horizon.

\section{Acknowledgments}

S.K was supported by the Basic Science Research Program through the NRF funded by the Ministry of Education (No. NRF-2014R1A1A2059080). B.H.L was supported by the National Research Foundation of Korea(NRF) grant funded by the Korea government(MSIP) No. 2014R1A2A1A01002306 (ERND).

\section{References}

1. A. H. Guth, Phys. Rev. D 23 (1981) 347. 
2. A. A. Starobinsky, Phys. Lett. B 91 (1980) 99. doi:10.1016/0370-2693(80)90670-X

3. A. D. Linde, Phys. Lett. B 108 (1982) 389.

4. A. A. Starobinsky, JETP Lett. 30 (1979) 682

5. B. Allen, Phys. Rev. D 37 (1988) 2078.

6. V. Sahni, Phys. Rev. D 42 (1990) 453.

7. P. A. R. Ade et al. [Planck Collaboration], arXiv:1502.02114 [astro-ph.CO].

8. V. A. Rubakov, M. V. Sazhin and A. V. Veryaskin, Phys. Lett. B 115 (1982) 189.

9. S. Koh, S. Kouwn, O. K. Kwon and P. Oh, Phys. Rev. D 88, 043523 (2013).

10. S. Koh, B. H. Lee and G. Tumurtushaa, arXiv:1512.05062 [gr-qc].

11. S. Kouwn, O. K. Kwon and P. Oh, Phys. Rev. D 91 (2015) 063521

12. C. Omero and R. Percacci, Nucl. Phys. B 165 (1980) 351.

13. M. Gell-Mann and B. Zwiebach, Phys. Lett. B 147 (1984) 111.

14. C. Armendariz-Picon, JCAP 0709 (2007) 014

15. S. Endlich, A. Nicolis and J. Wang, JCAP 1310 (2013) 011

16. J. Lee, T. H. Lee, T. Y. Moon and P. Oh, Phys. Rev. D 80 (2009) 065016

17. S. Koh, B. H. Lee and G. Tumurtushaa, arXiv:1512.05072 [gr-qc].

18. S. Chongchitnan and G. Efstathiou, Phys. Rev. D 73 (2006) 083511

19. W. Zhao, Chin. Phys. 16 (2007) 2894

20. L. A. Boyle and P. J. Steinhardt, Phys. Rev. D 77 (2008) 063504

21. M. S. Turner, M. J. White and J. E. Lidsey, Phys. Rev. D 48 (1993) 4613

22. W. Zhao and Y. Zhang, Phys. Rev. D 74 (2006) 043503 [astro-ph/0604458]. 